\title{
Inteligência emocional avaliada por autorrelato difere do construto personalidade?
}

\author{
Carla Woyciekoski - Universidade Federal do Rio Grande do Sul, Porto Alegre, Brasil \\ Claudio Simon Hutr ${ }^{1}$ - Universidade Federal do Rio Grande do Sul, Porto Alegre, Brasil
}

\begin{abstract}
Resumo
No presente estudo examinou-se a associação entre duas dimensões de personalidade, neuroticismo e extroversão, com inteligência emocional (IE), medida por meio de uma escala de Autorrelato. Participaram da pesquisa 131 pessoas, de ambos os sexos, com idades entre 18 e 49 anos. Os instrumentos utilizados foram a Escala Fatorial de Neuroticismo (EFN), a Escala Fatorial de Extroversão (EFEx) e a Medida de Inteligência Emocional (MIE). Análises correlacionais e de regressão múltipla demonstraram que as escalas de personalidade puderam explicar grande parte da variância da MIE. As dimensões de personalidade extroversão e neuroticismo, e a inteligência emocional medida pela MIE não se apresentaram como construtos independentes. Os resultados corroboraram uma série de estudos prévios e permitem inferir que não é adequado nem razoável medir a IE por meio de escalas de autorrelato.

Palavras-chave: Emoção, Inteligência emocional, Cognição, Personalidade, Avaliação.
\end{abstract}

Does emotional intelligence assessed by a Self-Report Scale differ from Personality?

\begin{abstract}
The present study examined the association between two personality dimensions, Neuroticism and Extraversion, with Emotional Intelligence (EI) assessed by a self-report measure. Participants were 131 men and women, aged between 18 and 49 years. The instruments used were the Factorial Neuroticism Scale (FNS), the Factorial Extraversion Scale (FES) and the Emotional Intelligence Measure (EIM). Correlational and Multiple Regression Analysis demonstrated that the personality scales could explain a large amount of the variance of the EIM. The personality dimensions, Extraversion and Neuroticism, and Emotional Intelligence, measured by self-report scales did not behave as independent constructs. These present results corroborated a series of previous studies, and allowed us to suggest that it is not adequate, nor reasonable to measure EI through self-reports.

Keywords: Emotion, Emotional intelligence, Cognition, Personality, Assessment.
\end{abstract}

As atuais concepções de inteligência refletem o pensamento de centenas de pesquisadores, que ao longo da história definiram o que significa ser inteligente. A literatura mostra que a inteligência tem sido definida como uma capacidade geral de compreensão e raciocínio ou como um conjunto de diversas capacidades mentais relativamente independentes umas das outras (Woyciekoski, 2006), gerando modelos que enfatizam fatores gerais ou específicos. Primi (2002) observou que a inteligência constitui um dos temas mais estudados na história da psicologia e destacou que a profusão de estudos e a complexidade do construto dificultam uma definição única. As definições tradicionais centram-se nas capacidades de raciocínio geral e de aprender a estabelecer relações (Spearman, 1923). Abordagens mais recentes introduziram novos elementos e capacidades, enfatizando os diversos contextos em que poderão ser aplicados (Gardner, 1995).

Os primeiros testes de inteligência foram desenvolvidos para predizer desempenho, atendendo a demandas nos contextos acadêmico e de seleção de pessoal (Roberts, Zeidner \& Matthews, 2001). Atentar

1 Endereço para correspondência:

Instituto de Psicologia, UFRGS

Rua Ramiro Barcelos, 2.600 - 90035-003 - Porto Alegre - RS.

E-mail: carwpsi@hotmail.com ou hutzc@terra.com.br para esse aspecto histórico pode ser relevante, pois as habilidades que integravam o comportamento considerado inteligente nesses contextos podem diferir das capacidades necessárias para atuação, adaptação e sucesso em novos tempos e ambientes, onde outras habilidades entram em cena, como as relacionadas ao mundo das emoções, criatividade e relacionamentos interpessoais (ver, por exemplo, Cobêro, Primi \& Muniz, 2006). Contudo, recentemente tem-se enfatizado a necessidade de incluir outras variáveis na predição de desempenho no trabalho, como aquelas que envolvem as capacidades relacionadas à compreensão emocional, resolução de problemas e tomada de decisão envolvendo emoções. No estudo conduzido por Cobêro e colaboradores (2006), a Inteligência Emocional foi um preditor do desempenho profissional, gerando informações adicionais e complementares às geradas por medidas tradicionais de inteligência.

A inteligência emocional (IE) constitui um campo de estudo que reúne pesquisas extensivas sobre a inter-relação entre pensamentos, sentimentos e habilidades. Sobretudo, investigam-se as reações e interpretações emocionais de sujeitos, bem como a função das emoções no comportamento inteligente. A consideração das interações entre cognição e emoção poderia resultar no reconhecimento da capacidade do homem em lidar com suas emoções de forma 
inteligente e compatível com seus mais caros objetivos de vida.

O estudo da IE permite ampliar o conceito do que é considerado como tradicionalmente inteligente, incluindo no espectro das inteligências aspectos relacionados às emoções e sentimentos. Essa área da pesquisa tem contribuído para reflexões críticas sobre a influência das emoções nos cenários clínico, educacional, ocupacional e social (Queroz \& Néri, 2005; Zeidner, Shani-Zinovich, Matthews \& Roberts, 2005).

A IE foi definida por Salovey e Mayer (1990) como uma habilidade mental, mais especificamente como uma subforma de inteligência social. Em 1997, Mayer e Salovey a definiram como

[...] a capacidade de perceber acuradamente, de avaliar e de expressar emocóes; a capacidade de perceber el on gerar sentimentos quando eles facilitam o pensamento; a capacidade de compreender a emoção e o conhecimento emocional; e a capacidade de controlar emoções para promover o crescimento emocional e intelectual. (p. 15)

Os autores propuseram um modelo de processamento de informações emocionais que envolve quatro capacidades: 1) percepção acurada das emoções; 2) uso da emoção para facilitar o pensamento, a criatividade e resolução de problemas; 3) compreensão das emoções; 4) gerenciamento emocional. Para avaliar essas capacidades, eles desenvolveram alguns instrumentos, sendo que o mais utilizado é o Mayer-Salovey-Caruso Emotional Intelligence Test (MSCEIT) (Mayer, Salovey \& Caruso, 2002). O teste é composto por oito tarefas que medem as quatro capacidades referidas. Percepção emocional é avaliada solicitando-se ao respondente que identifique emoções em faces e paisagens. $\mathrm{Na}$ facilitação de pensamento, o testando deve identificar que emoções promovem determinados pensamentos e atividades intelectuais. Compreensão emocional é medida por meio do entendimento de como as emoções se integram e se formam. Gerenciamento emocional é avaliado apresentando-se pequenas histórias que descrevem situações sociais e perguntando-se sobre o manejo das emoções nas respectivas situações. Segundo Mayer, Roberts e Barsade (2008), a IE reflete a habilidade de raciocinar sobre emoções, além de utilizá-las no processo do pensamento.

Mayer, Salovey e Caruso (2000) propuseram um modelo cognitivo, que define a IE por meio de habilidades e utiliza instrumentos de desempenho para avaliá-la. Outros autores incluíram, na sua definição de IE, habilidades não-intelectivas, como dimensões da personalidade, felicidade, tolerância ao estresse, caráter, impulsividade, competência social e pensamento criativo (Austin, Saklofske \& Egan, 2005; Bar-On,
1997; Goleman, 1996; Petrides \& Furnham, 2001; Saklofske, Austin \& Miniski, 2003; Schutte e cols., 1998; Tett, Fox \& Wang, 2005). Esses modelos teóricos foram denominados por Mayer e cols. (2000) de modelos mistos. Em geral, tais modelos utilizam questionários de autorrelato para acessar os componentes daquilo que definem por IE. Seus itens incluem frases como "Eu consigo expressar minhas necessidades na maior parte do tempo"; "Eu sou uma pessoa fácil de lidar e conviver". Mayer e cols. (2008) afirmaram que autorrelatos não possuem validade de conteúdo para acessar a IE, porque não medem raciocínio inteligente sobre emoções. Esses instrumentos não oferecem tarefas que permitam avaliar a capacidade dos sujeitos usarem as emoções e seu conhecimento emocional para promover pensamento e comportamento inteligente.

Até o momento, verificam-se dois métodos para avaliar a IE, um com instrumentos de autorrelato e outro com instrumentos de desempenho. Por meio dos últimos, mensura-se o desempenho do testando em tarefas específicas que envolvem a identificação de emoções, uso das emoções para produzir pensamento e resolver problemas. Nos primeiros, o participante é solicitado a reportar habilidades relativas à IE que acredita possuir. Autores argumentaram que esses dois métodos não medem o mesmo construto (Austin, Saklofske \& Egan, 2005; Jesus Junior \& Noronha, 2007; Mayer, Salovey \& Caruso, 2000; Van der Zee, Thijs \& Schakel, 2002).

Brackett e Mayer (2003) destacaram que as pessoas geralmente são incapazes de reportar acuradamente suas habilidades. Esses autores demonstraram uma fraca convergência entre escalas de desempenho e autorrelatos, com correlações de 0,18 e 0,21 entre o MSCEIT e o SSRI (Schutte Self-Report Inventory - Schutte et al., 1998), e o MSCEIT e o EQI (Emotional Quotient Inventory) (Bar-On, 1997), respectivamente. Brackett e cols. (2006 citados por Mayer e cols., 2008) desenvolveram uma escala de autorrelato baseada no seu modelo quádruplo da IE, e a correlacionaram com o MSCEIT, obtendo uma correlação de $r=0,19$ entre as duas escalas.

Além disso, escalas de autorrelato de IE em geral apresentam sobreposição com dimensões da personalidade. Em estudo realizado por Dawda e Hart (2000), o EQ-I correlacionou-se significativamente com quatro dimensões dos Cinco Grandes Fatores (CGF), e com depressão e alexitimia. Schutte e cols. (2001) encontraram correlações moderadas entre o SSRI e personalidade referente aos fatores alexitimia, otimismo, controle de impulso e abertura para experiência. A maioria das subescalas do EQ-I, do Multidimensional Emotional Intelligence Assessment e 
do SSRI correlacionam-se na faixa de $r=0,60$ a 0,70 com escalas de personalidade, como Neuroticismo dos CGF (Dawda \& Hart, 2000; Petrides \& Furnham, 2001; Tett, Fose \& Wang, 2005). Noutros estudos, as escalas dos CGF predisseram os escores do EQ-I apresentando correlações entre $r=0,75$ e 0,79 (Brackett \& Mayer, 2003; Grubb \& McDaniel, 2007).

Por outro lado, foram obtidas correlações mais elevadas entre escalas de habilidades cognitivas e o MSCEIT (Brackett \& Mayer, 2003; O'Connor \& Little, 2003). O Trait Meta Mood Scale (autorrelato) não se correlacionou com o Raven's Progressive Matrices $(\mathrm{r}=0,08)$, enquanto o MSCEIT obteve uma correlação de 0,27. No Brasil, Primi, Bueno e Muniz (2006) reportaram que as provas do MSCEIT discriminaram outras medidas de inteligência (como o BPR5) e medidas de personalidade (como o 16PF). Cobêro, Primi e Muniz (2006) encontraram correlações baixas entre o MSCEIT e o BPR5.

Visando contribuir para a discussão sobre os aspectos teóricos e de mensuração da IE, o objetivo deste estudo foi investigar se a Escala de Autorrelato MIE (Medida de Inteligência Emocional, de Siqueira, Barbosa \& Alves, 1999) mede alguma coisa que escalas de personalidade não medem. Os objetivos específicos foram examinar que proporção da variância dos escores obtidos na MIE poderiam ser explicados pela variância dos escores obtidos nas escalas de Extroversão e Neuroticismo. Além disso, foram analisadas as características psicométricas das escalas, a fim de compará-las com os resultados obtidos nos estudos originais.

\section{Método}

\section{Participantes}

Participaram deste estudo 42 homens e 89 mulheres matriculados em duas universidades do Rio Grande do Sul. A média de idade foi de 22, 7 anos (DP $=5,5)$. Os alunos eram oriundos dos cursos de Ciências Econômicas, Engenharia, Pedagogia, História, Jornalismo, Enfermagem e Psicologia.
Foram utilizadas duas escalas para acessar as dimensões de personalidade Neuroticismo e Extroversão. A Escala Fatorial de Neuroticismo (EFN) (Hutz \& Nunes, 2001) possui 82 itens em quatro subescalas: Vulnerabilidade, Desajustamento Psicossocial, Ansiedade e Depressão. A Escala Fatorial de Extroversão (EFEx) (Nunes \& Hutz, 2006) possui 57 itens em quatro subescalas: Comunicação, Altivez, Assertividade e Interação Social. Ambas as escalas demonstraram consistência interna e estrutura fatorial satisfatórias, validade convergente e boas correlações de teste-reteste (Hutz \& Nunes, 2001; Nunes \& Hutz, 2006). Os valores alfa de Cronbach do estudo original foram 0,95 (EFN) e 0,93 (EFEx). Para avaliar a IE utilizou-se a Medida de Inteligência Emocional (MIE) (Siqueira, Barbosa \& Alves, 1999), construída a partir do modelo de Goleman, com 59 itens distribuídos em cinco subescalas: Autoconsciência, Sociabilidade, Automotivação, Autocontrole e Empatia. Os coeficientes alfa de Cronbach do estudo original foram entre 0,78 e 0,87 .

\section{Procedimentos}

A presente pesquisa foi aprovada pelo Comitê de Ética da Universidade do Rio Grande do Sul. Os participantes foram recrutados nas salas de aula e convidados a participar de uma pesquisa sobre IE e personalidade. Após serem informados sobre o caráter voluntário de sua participação, coletou-se a assinatura do Termo de Consentimento Livre e Esclarecido e foi realizada a aplicação dos instrumentos.

\section{Resultados}

Inicialmente foram obtidas as médias, desvios padrão e consistências internas das cinco subescalas da MIE, as quais são apresentadas na Tabela 1. Nessa tabela também constam as mesmas informações relativas à EFN e EFEx. Esses resultados permitem comparar características psicométricas das escalas originais e na aplicação com as amostras deste estudo, e, como pode-se observar, praticamente não há diferenças.

\section{Instrumentos}

Tabela 1 - Média, desvio padrão e consistência interna da MIE, EFN e EFEx

\begin{tabular}{lccccc}
\hline Instrumento & Número de itens & Cronbach (Original) & Cronbach & Média & Desvio Padrão \\
\hline MIE & 59 & & 0,88 & 175 & 13,69 \\
Empatia & 14 & 0,87 & 0,85 & 40,9 & 4,48 \\
Sociabilidade & 13 & 0,82 & 0,83 & 35,3 & 5,08 \\
Automotivação & 12 & 0,82 & 0,85 & 37,8 & 4,73 \\
Autocontrole & 10 & 0,84 & 0,84 & 28,9 & 4,49 \\
Autoconsciência & 10 & 0,78 & 0,84 & 32,2 & 4,07 \\
EFN & 82 & 0,94 & 0,95 & 200,4 & 57,17 \\
\hline
\end{tabular}


Tabela 1. Média, Desvio Padrão e Consistência Interna da MIE, EFN e EFEx (Conclusão)

\begin{tabular}{lccccc}
\hline Instrumento & Número de itens & Cronbach (Original) & Cronbach & Média & Desvio Padrão \\
\hline Vulnerabilidade & 23 & 0,89 & 0,91 & 68,5 & 22,23 \\
Desajustamento & 14 & 0,82 & 0,80 & 23,5 & 9,68 \\
Ansiedade & 25 & 0,87 & 0,88 & 68,4 & 22,62 \\
Depressão & 20 & 0,87 & 0,90 & 40,1 & 16,92 \\
& & & & & \\
EFEx & 57 & & 0,93 & 254,6 & 57,17 \\
Comunicação & 19 & 0,90 & 0,91 & 86,2 & 21,49 \\
Altivez & 14 & 0,78 & 0,84 & 50,8 & 13,99 \\
Assertividade & 10 & 0,78 & 0,79 & 49,9 & 8,65 \\
Interação Social & 14 & 0,83 & 0,81 & 67,5 & 11,94 \\
\hline
\end{tabular}

Com o objetivo de examinar as relações entre sexo, idade e IE, e também para determinar se essas variáveis deveriam ser incluídas nas análises de regressão, foram produzidas correlações de Pearson. As correlações entre idade e IE foram próximas de zero $(r=0,04)$. Encontrou-se uma diferença de gênero significativa, porém pequena, entre homens $(M=171,5)$ e mulheres $(M=176,6) \quad(t=1,99 ; g l=129 ; \quad p<0,05$, $\mathrm{d}=0,37)$.

A seguir, foram realizadas correlações entre as dimensões Neuroticismo e Extroversão com IE. Os resultados são apresentados na Tabela 2. Como se pode ver nessa tabela, várias subescalas de Neuroticismo e, especialmente, de Extroversão apresentam correlações superiores a 0,40 com os escores das subescalas da MIE. Correlações dessa magnitude não são encontradas com frequência em estudos de personalidade na literatura. Deve-se também observar que, como seria esperado, tendo em vista as definições dos construtos, as correlações entre as subescalas de Neuroticismo e as da MIE são negativas.

Tabela 2 - Correlações entre os subfatores da MIE e os da EFN e da EFEx

\begin{tabular}{|c|c|c|c|c|c|c|c|c|c|c|}
\hline MIE & N1 & N2 & N3 & N4 & EFN & E1 & E2 & E3 & E4 & EFEx \\
\hline M1 & & & & & & 0,28 & 0,27 & & 0,40 & 0,37 \\
\hline M2 & $-0,38$ & & & $-0,44$ & $-0,35$ & 0,59 & 0,24 & 0,35 & 0,70 & 0,63 \\
\hline M3 & $-0,50$ & & $-0,25$ & $-0,61$ & $-0,50$ & 0,33 & 0,24 & 0,58 & 0,31 & 0,45 \\
\hline M4 & & $-0,29$ & & & & $-0,24$ & $-0,33$ & & & $-0,30$ \\
\hline M5 & & & & & & & & 0,23 & 0,34 & 0,30 \\
\hline MIE Total & & & & & $-0,39$ & & & & & 0,50 \\
\hline
\end{tabular}

Todas as correlações são significativas $(\mathrm{p}<0,01)$. Notas: MIE = Medida de Inteligência Emocional, M1 = Empatia, M2 = Sociabilidade, M3 = Automotivação, M4 = Autocontrole, M5 = Autoconsciência, MIE = Escore Total de Inteligência Emocional, N1 = Vulnerabilidade, N2 = Desajustamento Psicossocial, N3 = Ansiedade, N4 = Depressão, EFN = Escore Total de Neuroticismo, E1 = Comunicação, E2 = Altivez, E3 = Assertividade, E4 = Interação Social, EFEx = Escore Total de Extroversão.

Finalmente, foram realizadas análises de regressão múltipla com cada uma das cinco subescalas da MIE, utilizando cada um dos subfatores das escalas de Neuroticismo e Extroversão como preditores. As variáveis idade e sexo não foram incluídas nesse modelo em razão dos resultados anteriormente descritos. Nas análises de regressão, as variáveis foram incorporadas no modelo pelo método Stepwise. Os resultados são sumarizados na Tabela 3, onde se apresentam os subfatores das escalas de personalidade que entram nas equações para cada subescala da MIE.

\section{Discussão}

No presente estudo, as cinco subescalas da MIE apresentaram boa consistência interna, sendo que o coeficiente mínimo foi de 0,83 e o máximo de 0,85 . Esses resultados corroboraram os obtidos no estudo original (Siqueira e cols., 1999). O mesmo padrão de resultados foi obtido para EFN e EFEx. O coeficiente mínimo foi de 0,80 (EFN) e 0,79 (EFEx) e o coeficiente máximo foi de 0,91 para as duas escalas, respectivamente. 
Tabela 3 - Subescalas de Personalidade que são preditoras de inteligência emocional

\begin{tabular}{lcccc}
\hline \multicolumn{1}{c}{ Variável } & Beta & $\mathrm{R}$ & $\mathrm{R}^{2}$ & $\mathrm{p}<$ \\
\hline $\begin{array}{l}\text { Empatia (MIE1) } \\
\text { Interação social } \\
\quad \text { Sociabilidade (MIE2) }\end{array}$ & 0,40 & 0,40 & 0,16 & 0,01 \\
$\begin{array}{l}\text { Interação social } \\
\text { Comunicação }\end{array}$ & 0,53 & 0,70 & 0,49 & 0,01 \\
$\quad$ Automotivação (MIE3) & 0,29 & 0,74 & 0,54 & 0,01 \\
$\begin{array}{l}\text { Depressão } \\
\text { Assertividade }\end{array}$ & 0,41 & 0,61 & 0,37 & 0,01 \\
$\quad$ Autocontrole (MIE4) & 0,33 & 0,67 & 0,43 & 0,01 \\
$\begin{array}{l}\text { Altivez } \\
\text { Desajustamento }\end{array}$ & $-0,17$ & 0,33 & 0,11 & 0,01 \\
Comunicação & $-0,23$ & 0,37 & 0,14 & 0,01 \\
$\quad \begin{array}{l}\text { Autoconsciência (MIE5) } \\
\text { Interação social }\end{array}$ & $-0,19$ & 0,41 & 0,17 & 0,01 \\
$\quad$ & 0,34 & 0,34 & 0,12 & 0,01 \\
\hline
\end{tabular}

Pesquisadores vêm estudando a possibilidade das emoções interferirem no comportamento inteligente, por meio de sua influência nas reações das pessoas, e da forma que estas interpretam a informação emocional. Salovey e Mayer (1990) foram os primeiros a definir a IE através de habilidades baseadas num modelo psicométrico e hierárquico de inteligência. Eles propuseram que, para resolver problemas, pessoas devem identificar e monitorar emoções em si e nos outros, assim como integrar emoção e cognição para obter um melhor desempenho. Em contraste, modelos alternativos (BarOn, 1997; Goleman, 1996; Schutte e cols., 1998, Siqueira e cols., 1999) definiram a IE não apenas por meio de habilidades emocionais, mas também incluíram nas suas definições traços de personalidade, motivação e afeto. Esses modelos objetivam mensurar a IE por meio de questionários de autorrelato, os quais constituem medidas subjetivas da forma como indivíduos acreditam ser e comportar-se em relação as suas habilidades emocionais.

O principal objetivo do presente estudo foi verificar que proporção da variância da Escala de Autorrelato de IE, a MIE, poderia ser explicada por duas escalas de personalidade (EFN e EFEx). As análises de regressão demonstraram que essas duas escalas, em conjunto, explicaram $38 \%$ da variância da IE global medida pela MIE. Poder-se-ia argumentar que $62 \%$ da variância da MIE não foi explicada por estes instrumentos de personalidade e que, portanto, a MIE mede alguma coisa que não é acessada por estes instrumentos. Contudo, poder-se-ia também argumentar que foram utilizados, neste estudo, apenas dois $(40 \%)$ dos cinco fatores de personalidade e que não se deveria esperar uma explicação muito maior da que foi obtida.
O ponto importante, porém, é que dois fatores de personalidade explicam uma proporção elevada da variância da MIE, e esses resultados corroboram uma série de estudos prévios que demonstraram altas correlações entre questionários de autorrelato de IE e escalas de personalidade, incluindo Extroversão e Neuroticismo (Bracket \& Mayer, 2003; Ciarrochi, Chan \& Caputi, 2000; Dawda, \& Hart, 2000; Grubb \& McDaniel, 2007; Schutte e cols., 2001).

A análise dos resultados obtidos nas análises de correlação com as subescalas da MIE também mostra que vários domínios da IE são em grande parte explicados por características de personalidade. Por exemplo, com relação à Sociabilidade, $54 \%$ da variância desta subescala foi explicada apenas pelas subescalas Comunicação e Interação Social da EFEX. Siqueira e colaboradores (1999) referem Sociabilidade à "habilidade de iniciar e preservar as amizades, relacionar-se bem e ser aceito pelas pessoas, sentir-se bem entre elas [...]" (p. 149). Com base nessa definição e na forma como a MIE foi construída, não é possível afirmar que o subfator Sociabilidade, ou qualquer dos demais subfatores da MIE, estejam medindo habilidades intelectuais. A MIE é um questionário de autorrelato, por meio do qual os participantes são solicitados a responder perguntas relacionadas a capacidades que eles podem ou não possuir. $O$ fato de uma pessoa reportar possuir habilidades específicas não garante que ela de fato as possua. Talvez uma melhor definição para esse subfator poderia relacionarse ao quanto as pessoas acreditam possuir determinadas capacidades. Análises correlacionais e de regressão nos permitem inferir que há sobreposição de conceitos entre a forma como a IE é definida pelo modelo de Siqueira e colaboradores (1999) e neuroticismo e extroversão. 
Outra subescala da MIE que teve parte significativa de sua variância explicada pelas escalas EFN e EFEx foi Automotivação $\left(R^{2}=0,44\right)$. Foram obtidas correlações negativas significativas desse subfator com Vulnerabilidade $(r=-0,50)$, Ansiedade $(r=-0,25)$ e Depressão $(r=-0,61)$ e correlações positivas com Assertividade $(r=0,58)$, Comunicação $(r=0,33)$, Altivez $(r=0,24)$ e Interação Social $(r=0,31)$. Esses resultados eram esperados uma vez que altos escores em depressão podem refletir baixos níveis de expectativa em relação ao futuro, uma existência monótona, sem emoções. Como Hutz e Nunes (2001) observaram, pessoas depressivas reportam ser mais solitárias e ter dificuldades para formar objetivos e metas pessoais. A correlação positiva entre Assertividade e Automotivação também era esperada, pois a última reflete o quanto as pessoas acreditam serem assertivas e líderes, além de motivadas e ativas (Nunes, 2005). Sujeitos com altos escores em Vulnerabilidade podem revelar baixos níveis de persistência, insegurança e dependência de outros, apresentando dificuldades em constituir objetivos e metas pessoais independentemente de influência externa. Sujeitos ansiosos tendem a ser instáveis e reportar variabilidade de humor, irritabilidade e impulsividade (Hutz \& Nunes, 2001).

Siqueira e colaboradores (1999) relacionaram Automotivação com o nível de "persistência, coragem, força, otimismo e entusiasmo com que o indivíduo maneja objetivos e planos para a vida" (p. 150). Novamente, com base nessa definição e na forma como esse construto é acessado não é possível afirmar que essa subescala meça alguma capacidade. A definição desse subfator não abrange capacidades intelectuais, emocionais e/ou cognitivas. Os resultados encontrados podem sugerir que Automotivação, como Sociabilidade, possivelmente sejam variáveis mais relacionadas a características de personalidade do que decorrentes de habilidades da IE.

A dimensão interação social da EFEx explicou $16 \%$ da variância da subescala Empatia da MIE e foram ainda encontradas correlações baixas com outras duas subescalas (Comunicação e Altivez). De acordo com Siqueira e cols. (1999), esta faceta abrange um conjunto de itens relacionados à "habilidade" de identificar sentimentos, desejos, intenções, interesses e problemas nos outros, por meio da leitura e compreensão de elementos não verbais da comunicação. Embora as correlações entre as escalas de personalidade e a subescala Empatia da MIE tenham sido pequenas, algumas considerações sobre aspectos teóricos e de mensuração dessa última são pertinentes. Apesar da descrição da subescala Empatia e a primeira subescala do MSCEIT, Percepção
Emocional (PE), aparentemente apresentarem uma sobreposição conceitual, há uma diferença básica e crucial que deve ser considerada. Mayer e colaboradores (2002) descreveram a PE como a habilidade de identificar acuradamente distintas emoções em si e nos outros, assim como expressá-las em situações sociais. Eles propuseram que PE deveria ser medida por meio de desempenho em tarefas específicas, como a leitura de expressões faciais e de estados emocionais de personagens. Mesmo que Siqueira e colaboradores (1999) tenham definido Empatia como "um fator que congrega um conjunto de itens referentes à habilidade de identificar sentimentos, desejos [...]” (p. 149), esse fator não mede as habilidades dos sujeitos de identificar tais aspectos nos outros. Autorrelatos não requisitam dos respondentes a resolução de tarefas ou que decodifiquem expressões emocionais alheias (faciais ou de determinado personagem, por exemplo). Eles solicitam a autopercepção sobre capacidades. Questionar uma pessoa sobre suas capacidades emocionais não constitui forma plausível e adequada de mensurá-las.

Os subfatores altivez e comunicação (EFEx) e desajustamento psicossocial (EFN) explicaram 17\% $(R=0,41)$ da variável autocontrole. Siqueira e colaboradores (1999) definiram autocontrole como o nível de ponderação ante insultos, agressões, conflitos, sentimentos perturbadores e impulsos. Ao menos em tese, as correlações são plausíveis, pois sujeitos altos em desajustamento tendem a demonstrar níveis baixos de controle. Segundo Hutz e Nunes (2001), eles tendem a não respeitar regras sociais e a serem insensíveis ao sofrimento alheio, podendo envolver-se em sérias violações. A análise dos itens do subfator altivez demonstrou que pessoas altas nessa dimensão tendem a superestimar seu valor e capacidade, demonstrando maior preocupação com o poder $\mathrm{e}$ influência sobre os outros, não parecendo priorizar o controle de seus impulsos. Contudo, aparentemente, essa subescala não mede capacidade de controle emocional.

Interação social (EFEx) explicou $12 \%$ da variância da subescala Autoconsciência. Siqueira e colaboradores (1999) descreveram que autoconsciência reflete ações introspectivas de reconhecimento, codificação, avaliação e identificação de sentimentos. As correlações obtidas são plausíveis na medida em que maior nível de autoconsciência pode refletir melhor condição para relacionar-se com outros e desempenhar funções de liderança, associadas à assertividade. Porém, os itens não medem capacidades intelectuais. 


\section{Considerações finais}

Os resultados do presente estudo demonstraram uma importante sobreposição dos subfatores da MIE com os subfatores de Neuroticismo e Extroversão. Os dados corroboram estudos prévios (Dawda \& Hart, 2000; Saklofske, Austin \& Miniski, 2003; Van der Zee, Thijs \& Schakel, 2002) que demonstraram sobreposição de Neuroticismo e Extroversão com autorrelatos de IE. Esses achados parecem indicar que a MIE não se distingue de escalas de personalidade e não constitui uma medida independente de IE.

Embora o objetivo da presente pesquisa não fosse questionar a viabilidade da IE enquanto um construto psicológico, discutiu-se a forma como ela tem sido conceituada e mensurada. Esses são aspectos a serem levados em conta na investigação de uma nova forma de inteligência. Sabe-se que a viabilidade, utilidade e relevância da IE dependem de sua capacidade de constituir-se como um novo construto psicológico distinto e independente de outros preexistentes. Ela deve medir e predizer importantes aspectos da vida e comportamento humano, os quais não tenham sido captados por medidas anteriormente desenvolvidas (Brackett \& Mayer, 2003). O aspecto problemático do uso de escalas de autorrelato para medir a IE refere-se ao fato de que se baseiam na autopercepção das pessoas sobre as suas capacidades emocionais, e esta com frequência não corresponde às capacidades reais (Zeidner e cols., 2005). Portanto, não constituem uma forma adequada de medi-la. Faz-se necessária a avaliação do desempenho do indivíduo por meio de tarefas específicas que lhe permitam emitir respostas ou comportamentos, a fim de que suas reais capacidades sejam acessadas e avaliadas.

Os testes de IE de desempenho desenvolvidos pelo modelo cognitivo, tais como o MSCEIT, constituem as medidas de IE mais promissoras, pois têm se correlacionado com escalas de inteligência (como as medidas de QI). Assim como Zeidner e colaboradores (2005) postularam, o futuro da investigação da IE está fundamentalmente ancorado no desenvolvimento e aprimoramento dessas escalas. Há evidências de que o MSCEIT e seu predecessor MEIS acessam algo que nunca havia sido medido antes, ou seja, uma nova forma de inteligência, a IE (Brackett \& Mayer, 2003; Mayer e cols., 2002; Roberts e cols., 2001).

Embora autorrelatos não constituam medidas adequadas da IE, podem ser úteis para acessar o que denominou-se de IE autorreferida (Mayer e cols., 2004), pois baseiam-se nas percepções que as pessoas têm de suas emoções, habilidades e controle emocional. Poderiam gerar medidas de autopercepção da IE. Porém, conforme Mayer e colaboradores (2004) e Zeidner e colaboradores (2005), as pessoas raramente têm consciência de suas habilidades emocionais. Esse padrão de correlação é notório e merece futuras investigações. Em virtude da ausência de uma escala de desempenho de IE publicada e validada para a população brasileira no momento desta pesquisa, essas correlações não foram examinadas.

Por fim, objetivou-se também, com este trabalho, incitar teóricos e pesquisadores a participarem da discussão cada vez mais atual da necessidade de ampliação do que tem sido considerado tradicionalmente como inteligência ou comportamento inteligente. São muitos os trabalhos que estabelecem a natureza de suas interfaces com aspectos orgânicos, emocionais, educacionais, psicológicos e culturais (BarOn, 1997; Gardner, 1995; Goleman, 1996; Mayer e cols., 2004; Queroz \& Néri, 2005; Schutte e cols., 2001). Argumenta-se que os modelos clássicos não consideraram a complexidade do funcionamento humano; pois a inteligência não reflete apenas a capacidade para resolver problemas acadêmicos e laborais, mas também a de agir de forma estratégica e inteligente nos contextos emocionais, pessoais e sociais. Sendo assim, a IE oferece a possibilidade de incluir no âmbito das inteligências existentes as relações entre cognição e emoção, assim como os seus efeitos e impacto no comportamento adaptativo e no bem-estar dos sujeitos.

\section{Referências}

Austin, E. J., Saklofske, D. H. \& Egan, V. (2005). Personality, well-being and health correlates of trait emotional intelligence. Personality and Individual Differences, 38, 547-558.

Bar-On, R. (1997). The emotional intelligence inventory (EQI): technical manual. Toronto: Multi-Health Systems.

Brackett, M. A. \& Mayer, J. D. (2003). Convergent, discriminant, and incremental Validity of Competing Measures of Emotional Intelligence. Personality and Social Psychology Bulletin, 9, 11471158.

Ciarrochi, J., Chan, A. \& Caputi, P. (2000). A critical evaluation of the emotional intelligence construct. Personality and Individual Differences, 28, 539-561.

Cobêro, C., Primi, R. \& Muniz, M. (2006). Inteligência emocional e desempenho no trabalho: um estudo com MSCEIT, BPR-5 e 16PF. Paideia, 16, 337348. 
Dawda, D. \& Hart, S. D. (2000). Assessing emotional intelligence: reliability and validity of the Bar-On Emotional Quotient Inventory (EQ-i) in university students. Personality and Individual Differences, 28, 797-812.

Gardner, H. (1995). Inteligências múltiplas: a teoria na prática. (M. A. V. Veronese, Trad.). Porto Alegre, Brasil: Artes Médicas. (Original publicado em 1993).

Goleman, D. (1996). Inteligência emocional. (M. Santarrita, Trad.). Rio de Janeiro, Brasil: Objetiva. (Original publicado em 1995).

Grubb, W. L. \& McDaniel, M. A. (2007). The fakability of Bar-On's emotional quotient inventory short form: catch me if you can. Human Performance, 20, 43-59.

Hutz, C. S. \& Nunes, C. H. S. S. (2001). Escala Fatorial de Neuroticismo. São Paulo: Casa do Psicólogo.

Jesus Junior, A. G. \& Noronha, A. P. P (2007). Inteligência emocional e provas de raciocínio: um estudo correlacional. Psicologia: Reflexão e Crítica, 20(3), 480-489.

Mayer, J. D. \& Salovey, P. (1997). What is emotional intelligence? Em P. Salovey \& D. Sluyter (Orgs.). Emotional development and emotional intelligence: implications for educators (pp. 3-31). Nova Iorque, USA: Basic Books.

Mayer, J. D., Salovey, P. \& Caruso, D. R. (2000). Models of emotional intelligence. Em R. J. Sternberg (Orgs.). Handbook of Intelligence (pp. 396420). Nova Iorque: Cambridge.

Mayer, J. D., Salovey, P. \& Caruso, D. R. (2002). Mayer-Salovey-Caruso Emotional Intelligence Test (MSCEIT) user's manual. Toronto, Ontario, Canada: MHS Publishers.

Mayer, J. D., Salovey, P. \& Caruso, D. R. (2004). A further consideration of the issues of emotional intelligence. Psychological Inquiry, 15(3), 249-255.

Mayer, J. D., Roberts, R. D. \& Barsade, S. G. (2008). Human abilities: emotional intelligence. Annual Review of Psychology, 59, 507-536.

Nunes, C. H. S. S. (2005). Construção, normatização e validação das escalas de socialização e extroversão no modelo dos cinco grandes fatores. Tese de Doutorado. Programa de Pós-Graduação em Psicologia. Universidade Federal do Rio Grande do Sul Porto Alegre, RS.

Nunes, C. H. S. S. \& Hutz, C. S. (2006). Escala Fatorial de Extroversão. São Paulo: Casa do Psicólogo.
O’Connor, R. M. Jr. \& Little, I. S. (2003). Revisiting the predictive validity of emotional intelligence: self-report versus ability-based measures. Personalility and Individual Differences, 35(8), 18931902.

Petrides K. V. \& Furnham, A. (2001). Trait emotional intelligence: psychometric investigation with reference to established trait taxonomies. European Journal of Personality, 15, 425-448.

Primi, R. (2002). Inteligência fluida: definição fatorial, cognitiva e neuropsicológica. Paidéia RibeirãoPreto), 12(23), 57-75.

Primi, R., Bueno, J. M. H. \& Muniz, M. (2006). Inteligência emocional: validade convergente $\mathrm{e}$ discriminante do MSCEIT com a BPR5 e o 16PF. Psicologia Ciência e Profissão, 26(1), 26-45.

Queroz, N. C. \& Néri, A. L. (2005). Bem-estar psicológico e inteligência emocional entre homens e mulheres na meia-idade e velhice. Psicologia: Reflexão e Crítica, 18(2), 292-299.

Roberts, R. D., Zeidner, M. \& Matthews, G. (2001). Does emotional intelligence meet traditional standards for an intelligence? Emotion, 1, 196-231.

Saklofske, D. H., Austin, E. J. \& Miniski, P. S. (2003). Factor structure and validity of $\mathbf{a}$ trait emotional intelligence measure. Personality Individual Differences, 34, 707-721.

Salovey, P. \& Mayer, J. D. (1990). Emotional intelligence. Imagination, Cognition and Personality, 9, 185-211.

Schutte, N. S., Malouff, J. M., Hall, L. E., Haggerty, D. J., Cooper, J. T., Golden, C. J. \& Dornheim, L., (1998). Deveolpment and validation of a measure of emotional intelligence. Personality and Individual Differences, 25, 167-177.

Schutte, N. S., Malouff, J. M., Bobik, C., Coston, T. D., Greeson, C., Jedlicka, C., Rhodes, E. \& Wendorf, G. (2001). Emotional intelligence and interpersonal relations. Journal of Social Psychology, 141, 523-536.

Siqueira, M. M. M., Barbosa, N. C. \& Alves, M. T. (1999). Construção e validação fatorial de uma Medida de Inteligência Emocional. Psicologia: Teoria e Pesquisa, 15, 143-152.

Spearman, C. (1923). The Nature of intelligence and the principles of cognition. Londres: Macmillan.

Tett, R. P., Fox, K. E. \& Wang, A. (2005). Development and validation of a self-report measure of emotional intelligence as a 
multidimensional trait domain. Personality Society of Psychology Bulletin, 31, 859-888.

Van der Zee, K., Thijs, M. \& Schakel, L. (2002). The relationship of emotional intelligence with academic intelligence and Big Five. European Journal of Personality, 16, 103-125.

Woyciekoski, C. (2006). Instrumentos de inteligência emocional de autorrelato medem alguma coisa que instrumentos de personalidade não medem? Dissertação de Mestrado. Programa de Pós-
Graduação em Psicologia. Universidade Federal do Rio Grande do Sul - Porto Alegre, RS.

Zeidner, M., Shani-Zinovich, I., Matthews, G. \& Roberts, R. D. (2005). Assessing emotional intelligence in gifted and non-gifted high school students: outcomes depend on the measure. Intelligence, 33, 369-391.

Recebido em dezembro de 2009

Reformulado em marco de 2010 Aprovado em maio de 2010

Sobre os autores:

Carla Woyciekoski possui graduação em Psicologia pela Universidade de Santa Cruz do Sul (1999) e mestrado em Psicologia pela Universidade Federal do Rio Grande do Sul (UFRGS, 2006). Atualmente é doutoranda em Psicologia na UFRGS. É membro em Formação em Psicanálise na Instituição Psicanalítica Centro de Estudos Lacaneanos em Porto Alegre.

Claudio Simon Hutz possui graduação em Psicologia pela Universidade de Haifa (1973), mestrado e doutorado em Psicologia pela University of Iowa 1979, 1981. Atualmente é professor titular da Universidade Federal do Rio Grande do Sul. Foi presidente da ANPEPP e do IBAP e participou em comissões da CAPES, CNPQ, FAPERGS e FAPESP. Foi chefe de Departamento, coordenador do Programa de PG em Psicologia e diretor do Instituto de Psicologia da UFRGS. 